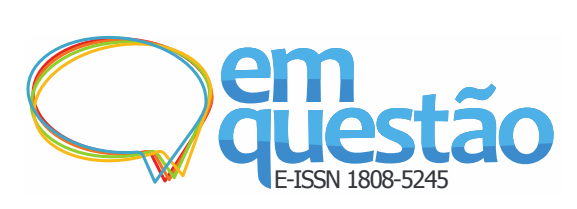

\title{
Indicativos do campo científico: análise da produtividade dos bolsistas PQ 1 e PQ 2 em Comunicação (2004-2013)
}

\author{
Willian Lima Melo \\ Mestre; Universidade Federal de Alagoas, Delmiro Gouveia, AL, Brasil; \\ willianmelo23@gmail.com \\ Anna Elizabeth Galvão Coutinho Correia \\ Doutora; Universidade Federal de Pernambuco, Recife, PE, Brasil; \\ aegcc3@gmail.com
}

\begin{abstract}
Resumo: São apresentados levantamentos referentes à produtividade dos 124 bolsistas PQ em Comunicação. O objetivo proposto foi relacionar as 7.820 produções dos bolsistas de produtividade em pesquisa 1 e 2 no período 20042013 à caracterização do campo científico da área de Comunicação no Brasil. A pesquisa é de cunho exploratório qualitativo e foi utilizado o emparelhamento (pattern matching) como estratégia auxiliar de análise. Os indicativos alcançados ajudaram a interpretar elementos do campo científico da Comunicação. Constatou-se que a composição desse campo científico específico é derivada de relações sociais dos agentes participantes, bem como do uso de ações de conhecimento e reconhecimento de suas respectivas produções, reforçando o caráter não fortuito dos atos dos agentes e instituições.
\end{abstract}

Palavras-chave: Comunicação; Bolsistas de Produtividade; Ensino Superior Pesquisa; Sociologia do Conhecimento.

\section{Introdução}

O presente estudo é tido como continuação dos estudos iniciados, desenvolvidos e apresentados por Melo (2015) e Melo e Correia (2015) no Programa de PósGraduação em Ciência da Informação da Universidade Federal de Pernambuco (PPGCI-UFPE), no Encontro Nacional de Pesquisa e Pós-Graduação em Ciência da Informação (XVI ENANCIB, 2015) e no Encontro Brasileiro de Bibliometria e Cientometria (5 $\left.5^{\circ} \mathrm{EBBC}, 2016\right)$. Propõe discussões sobre a política nacional de incentivo à produção científica no país, problematizando seu funcionamento e consequências para um campo social específico, o científico.

Inicialmente, trabalhando com a produtividade científica dos bolsistas PQ 1 em Comunicação (MELO, 2015; MELO; CORREIA, 2015), entendeu-se a 
necessidade de expansão do estudo dessa temática específica. Com isso, situa-se aqui, junto com as 3.548 produções dos 53 bolsistas PQ 1, as 4.272 produções científicas dos 71 bolsistas PQ 2 em Comunicação como objeto de estudo.

É uma pesquisa de cunho exploratório, na qual foi percebida a recorrência a métodos quantitativos para a organização da informação. Por esse motivo, recorre-se ao debate sobre a aplicabilidade da estratégia metodológica do emparelhamento (pattern matching) ao estudo, na tentativa de atingir melhores resultados de análises qualitativas. Sobre o problema, é vista a questão da possibilidade de se obter indícios sobre o campo científico da área da Comunicação, por meio da análise comparativa da produção científica dos bolsistas PQ 2.

O quadro teórico utilizado serve de auxílio na percepção mais crítica da produção e produtividade científica no Brasil, pois apresenta discussões que versam sobre a Ciência (MERTON, 2013; BOURDIEU, 2008), campo científico (BOURDIEU, 2004; SHINN; RAGOUET, 2008) e a política científica brasileira (MOREL, 1979; SOCIEDADE BRASILEIRA PARA O PROGRESSO DA CIÊNCIA, 2011).

As análises apresentadas já conseguem incitar o debate sobre a atual situação política nacional de incentivo à produção científica de alto nível. E sobre o tratamento das informações, é possível inferir sobre a aplicabilidade do método do emparelhamento, que foi utilizado na verificação de padrões dos agentes presentes no campo científico.

\section{Referencial teórico}

A natureza da Ciência é inerentemente social, estabelece dependências para emergir antes e depois de seu desenvolvimento. Como afirma Melo, a Ciência pode ser interpretada como informação que

[...] não expressa somente a capacidade individual, ou coletiva, de organização do conhecimento do indivíduo. Ela também exprime a intencionalidade cognitiva do homem que a quer ver representada em seu meio. (MELO, 2015, p. 43). 
No campo da Ciência, a busca pela representatividade é alcançada respeitando normas específicas, o que Merton (2013) chama de quintessência da Ciência moderna. Sintetizando A ciência e a estrutura social democrática, artigo de autoria de Merton no ano de 1942, Shinn e Ragouet (2008) descrevem as quatro normas necessárias para o alcance do éthos científico: o universalismo; o comunismo; o desinteresse; e o ceticismo organizado.

Shinn e Ragouet (2008, p. 20) expõem que o imperativo do Universalismo “[...] está ligado à aplicação de critérios impessoais preestabelecidos”; e indica que "[...] aceitação e rejeição de proposições científicas não poderiam estar subordinadas à apreciação dos atributos pessoais ou sociais de seus produtores". Sobre o Comunismo, o entendimento deve ser de que "a ciência é uma atividade pública que leva a produção coletiva de bens públicos. [...] A retribuição que um cientista obtém por ter produzido resultados válidos é um reconhecimento público". O Desinteresse não é sinônimo de altruísmo; motivações pessoais ou extracientíficas podem existir, ou seja, “[...] os cientistas são honestos, mas essa honestidade está, antes de tudo, ligada ao exercício de um controle público que se poderia qualificar de intersubjetivo". Por último, o ceticismo organizado revela a preocupação dos cientistas em “[...] não se deixar influenciar por suas convicções pessoais quando avaliam os trabalhos de seus colegas".

Por vezes os estudos mertonianos são considerados genéricos (MOREL, 1979), e, nesse sentido, as contribuições de Bourdieu (2004; 2008) podem ser interpretadas de forma complementar. O referido autor analisa a Ciência, o seu complexo funcionamento, os respectivos atores sociais e os resultados científicos de maneira austera, porém problematizada e de fácil analogia à realidade.

A discussão de Bourdieu (2008) sobre o campo científico diz respeito a um espaço de conquista e perda de poder. Um ambiente mutável de característica fragmentada e desproporcional, não eventualmente, mas sim devido ao acúmulo de capital científico dos agentes e instituições presentes nesse campo.

A literatura referente à Ciência presente em Merton e Bourdieu não se excluem - ao contrário, demonstram perspectivas distintas e complementares de uma temática complexa. Shinn e Ragouet (2008) reconhecem a importante 
contribuição de Bourdieu para os estudos sobre a Ciência com o desenvolvimento do conceito de campo científico. Neste trabalho, cabe ressaltar, como lembra Bourdieu, que

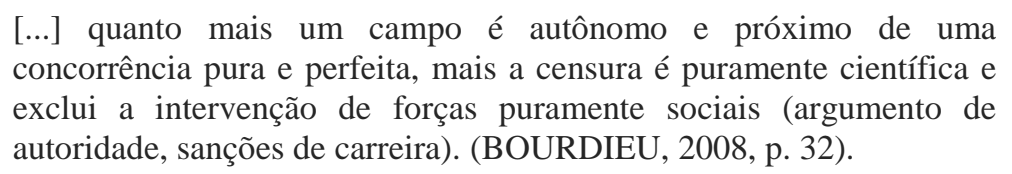
concorrência pura e perfeita, mais a censura é puramente científica e exclui a intervenção de forças puramente sociais (argumento de autoridade, sanções de carreira). (BOURDIEU, 2008, p. 32).

Com a relativa autonomia de um campo é possível observar o fator de o campo científico manter relações com outros campos sociais (o econômico e o político, por exemplo), criando uma relação de mutualismo entre os mesmos, em que os resultados sejam benéficos para os envolvidos. Vale lembrar que a prática científica não é uma atividade de ínfimo custeio - muitas vezes, subsídios financeiros são necessários para o alcance de resultados.

Nesse sentido, ganha sentido a política científica de auxílios exercida pelo Brasil por meio de suas instituições. Este estudo dedica atenção ao Conselho Nacional de Desenvolvimento Científico e Tecnológico - CNPq (2006).

Criado em 1951, o CNPq desempenha atividades relativas à formulação e condução das políticas de Ciência, Tecnologia e Inovação. O Programa de Bolsa de Produtividade (PQ) é indicado pela Sociedade Brasileira para o Progresso da Ciência (SOCIEDADE BRASILEIRA PARA O PROGRESSO DA CIÊNCIA, 2011) como um dos responsáveis pelo desenvolvimento da Pós-Graduação Brasileira. Segundo Melo (2015, p. 52), é “[...] responsável pelo fomento, que visa aumentar/melhorar a produtividade dos pesquisadores selecionados".

A execução do programa PQ é uma continuação da máxima descrita por Morel (1979, p. 23, grifo do autor), que, ao analisar o $\mathrm{CNPq}$, descreve que “ciência e recursos humanos vão ser valorizados como fatores de progresso, elementos fundamentais para o aprimoramento das forças produtivas e a expansão capitalista".

Existem no Brasil, aproximadamente, 14.500 bolsistas de produtividade de todas as áreas do conhecimento distribuídos nas categorias PQ 1 (1A, 1B, 1C, 1D) e PQ 2. Este trabalho dedicou atenção aos 124 bolsistas PQ 1 e 2 da área de Comunicação. 
Seguindo o raciocínio descrito por Bourdieu (2008), sendo entendido como elo de comunicação e satisfação mútua entre o campo científico e o campo político/econômico, o Programa de Bolsa de Produtividade altera características de concorrência puras e perfeitas presentes em um campo. A característica da necessidade por representatividade científica (ganho de capital científico) se faz presente, suscitando padrões que podem ser observados entre os agentes componentes do campo, fator que pode ser percebido com a aplicação da metodologia do emparelhamento.

\section{Procedimentos e metodologia de análise}

Existe na Plataforma Sucupira (Coordenação de Aperfeiçoamento de Pessoal de Nível Superior - CAPES) a indicação dos Programas de Pós-Graduação reconhecidos e recomendados na área de avaliação das Ciências Sociais Aplicadas I (Ciência da Informação; Comunicação; Desenho Industrial; e Museologia).

Com relação ao Programa de Produtividade em Pesquisa do CNPq, encontra-se, na área acima descrita, a distribuição de 124 bolsas PQ para Comunicação; 49 bolsas para Ciência da Informação; 33 para Desenho Industrial; e apenas cinco bolsas de produtividade para Museologia.

Demonstrou-se interesse particular pela área da Comunicação inicialmente pelo quantitativo de bolsas existente (maior do que as demais áreas); outro detalhe diz respeito à obtenção de conhecimento de indícios do campo científico da área (Ciências Sociais Aplicadas I), por nela os autores estarem inseridos. Vale lembrar ainda que este trabalho encerra uma análise realizada anteriormente sobre a produtividade científica dos bolsistas PQ 1 em Comunicação (MELO, 2015; MELO; CORREIA, 2015). Encontram-se aqui, neste estudo, uma análise sobre 7.820 produções de 124 bolsistas de produtividade PQ 1 e PQ2.

A pesquisa foi desenvolvida respeitando os seguintes procedimentos: (1) levantamento e revisão da leitura exploratória; (2) identificação dos bolsistas de produtividade PQ 2 em Comunicação; (3) levantamento dos Currículos Lattes e detalhamento das informações dos 124 bolsistas PQ 1 e PQ 
2 em Comunicação; (4) tratamento qualitativo dos dados encontrados; (5) tabulação dos resultados.

Após o levantamento dos Currículos Lattes, foram detalhadas informações sobre o quantitativo de bolsas de produtividade em pesquisa, a formação acadêmica dos pesquisadores PQ, a Instituição de Ensino Superior (IES) a que eles estão vinculados e a produtividade científica de livros, capítulos de livros, artigos em periódicos científicos e trabalhos contidos em anais de congressos.

Qualitativamente, o tratamento das informações seguiu o raciocínio dos aportes teóricos sugeridos para análise. Ou seja, tentou-se um tratamento que teve por objetivo a interpretação de possíveis ganhos, perdas ou estagnação de capital simbólico entre os bolsistas analisados. Realizou-se, por exemplo, a identificação de variáveis dentro de algumas características levantadas: sobre o quantitativo de bolsas, verificou-se a distribuição do quantitativo de bolsas por regiões; sobre a formação acadêmica, verificou-se se o pesquisador possuía estágio pós-doutoral; sobre a IES a que os bolsistas estão vinculados, verificou-se se a IES do pesquisador era pública ou privada; sobre a produtividade científica, verificou-se a produtividade por região isoladamente e organizou-se o quantitativo das publicações de artigos publicados em periódicos, bem como o respectivo estrato Qualis dessas revistas.

Vale ressaltar ainda que o grupo PQ 1 e o grupo PQ 2 de pesquisadores foram analisados, em início, isoladamente, e que, após o término dos procedimentos de análise citados acima, foi realizado o procedimento de análise comparativa dos resultados desses dois grupos.

Informações sobre a produção científica realizada por este grupo, entre os anos de 2004 a 2013, foram resgatadas na Plataforma Lattes do CNPq, utilizando a ferramenta do Currículo Lattes. Como indicado anteriormente, o atual trabalho é a continuação de trabalhos anteriores; os levantamentos dos dados foram realizados nas seguintes datas: bolsistas PQ 1 - 11 set 2014; e bolsistas PQ $2-8$ ago 2015. A descontinuidade temporal entre um levantamento e outro é justificada devido ao desenvolvimento de trabalhos com perspectivas específicas (MELO; CORREA, 2015; MELO; CORREA, 2016), 
porém aqui existe a característica do resgate e cruzamento de informações, trazendo novos indícios de leitura de parte do campo científico da Comunicação.

Este é um estudo de características exploratórias em que são usadas modalidades de análises qualitativas. É apontado o emparelhamento (pattern matching) como estratégia para o alcance das apreciações. É válido neste trabalho o argumento proposto por Laville e Dionne (1999, p. 225), no qual é visto que perspectivas qualitativas e quantitativas não são opostas e sim "[...] podem até parecer complementares, cada uma ajudando à sua maneira o pesquisador a cumprir sua tarefa, que é extrair as significações essenciais da mensagem".

A escolha do emparelhamento como estratégia auxiliar de análise tem por base, segundo Laville e Dionne (1999, p. 227), a realização de uma associação dos dados encontrados a um modelo teórico com a finalidade de estabelecer comparações, ou seja, é uma estratégia que supõe “[...] a presença de uma teoria sobre a qual o pesquisador apoia-se para imaginar um modelo do fenômeno ou da situação em estudo".

Reforçando a utilização de metodologias com esse perfil é possível encontrar a proposição de que

[...] os diversos sistemas de representação e notação inventados pelo homem ao longo dos séculos têm por função semiotizar, reduzir a uns poucos símbolos ou a alguns poucos traços os grandes novelos confusos da linguagem, sensação e memória que formam o nosso real. [...] Talvez a combinação destas duas características, o dom da manipulação e a imaginação, possa explicar o fato de que quase sempre pensemos com o auxílio de metáforas, de pequenos modelos concretos, muitas vezes de origem técnica. (LÉVY, 1993, p. 70).

Traduzir uma realidade/lógica abstrata, como a do campo científico, não é simples, e, nesse sentido, o método do emparelhamento é eficiente. É conveniente frisar que esta pesquisa tem aspectos multidisciplinares e reconhece na área da Ciência da Informação a potencialidade de alcance dos objetivos pretendidos. Guimarães, Gracio e Matos (2014) justificam que por meio de estudos semelhantes a este, é possível identificar, evidenciar e visualizar a distribuição científica dos bolsistas de produtividade, levando em consideração a relevância e 
contribuição das pesquisas geradas pelos pesquisadores para o desenvolvimento da Ciência.

\section{Resultados e discussões}

Foi possível observar no desenvolvimento do trabalho alguns indícios de composição e funcionamento do campo científico em que os 124 bolsistas PQ estão inseridos. São dados de abrangência regional e nacional que conseguem revelar disparidades relativas à distribuição de bolsistas e à produtividade, aos tipos e destinações das produções, por exemplo.

Os resultados traduzem informações sobre o perfil dos pesquisadores estudados; as características sobre as instituições de ensino superior a que estes estão vinculados; a formação acadêmica desses profissionais; e também os números brutos das produções científicas (capítulos de livro; artigos em periódicos; trabalhos em anais de congresso; livros).

O Gráfico 1 indica a formação acadêmica dos bolsistas PQ em Comunicação no Brasil. A verificação da conclusão de estágio pós-doutoral é uma característica presente nos dois grupos (PQ 1 e PQ 2). Vale ressaltar que o estágio pós-doutoral não confere ao pesquisador um grau de titulação, como é o de mestre e/ou doutor, por exemplo, mas sim uma indicação de continuação aprimorada da atividade de pesquisa. Em outras palavras, mesmo não carregando o peso de uma titulação, a experiência do estágio de pós-doutoramento consegue inserir em um determinado campo um reconhecimento diferenciado, podendo ser interpretado, como afirma Bourdieu (2004), um tipo específico de capital científico. 
Gráfico 1 - Comparação entre os bolsistas PQ 1 e PQ 2: formação acadêmica.

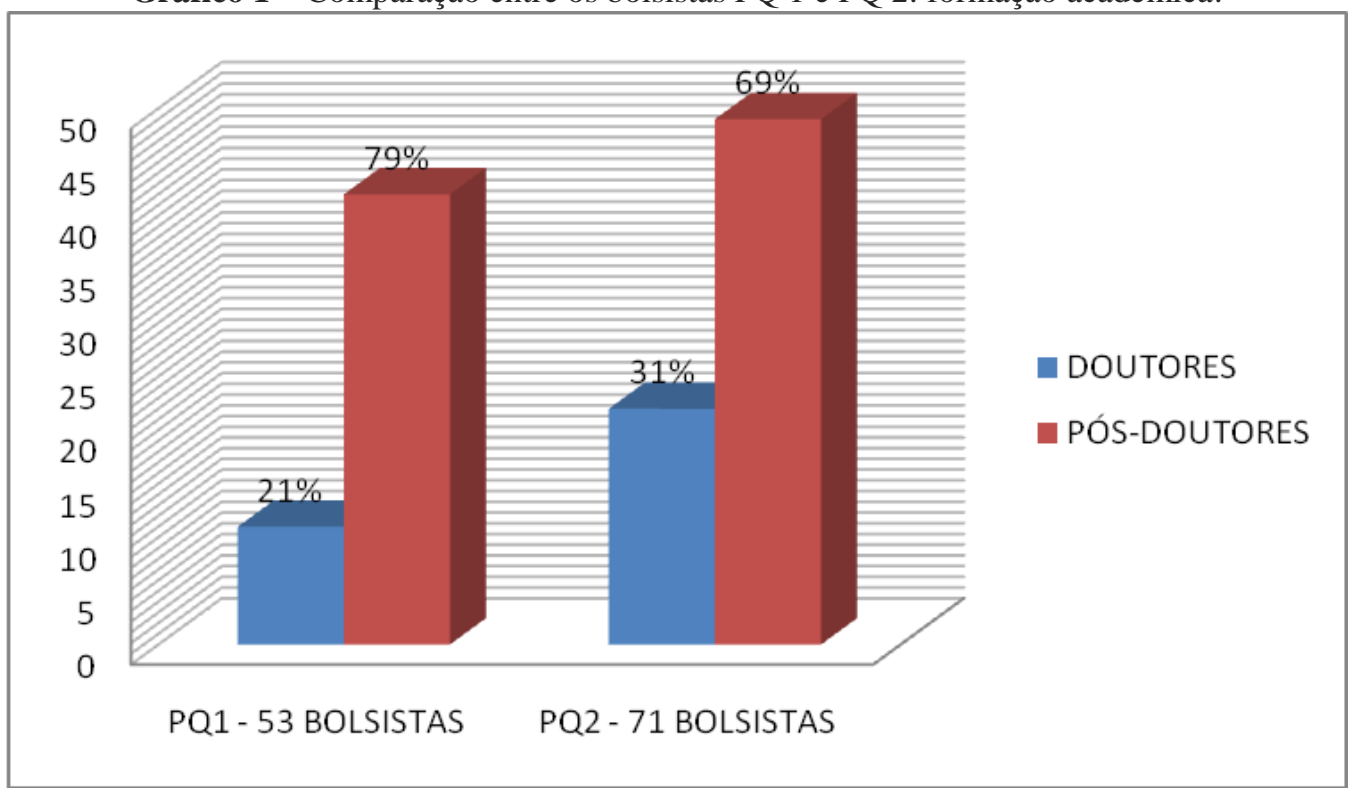

Fonte: Elaborado pelos autores.

O Gráfico 2 traz informações relativas à distribuição dos 124 bolsistas PQ pelo Brasil. A região Norte não é evidenciada neste levantamento. Sobre as demais regiões, é possível perceber a seguinte distribuição: Centro-Oeste três bolsistas (CO: DF; GO); Nordeste - 14 bolsistas (NE: BA; PB; PE; RN); Sudeste - 73 bolsistas (SE: MG; RJ; SP); e Sul - 34 bolsistas (S: PR; SC; $\mathrm{RS})$.

Gráfico 2 - Distribuição por região e instituições e vínculo dos bolsistas PQ.

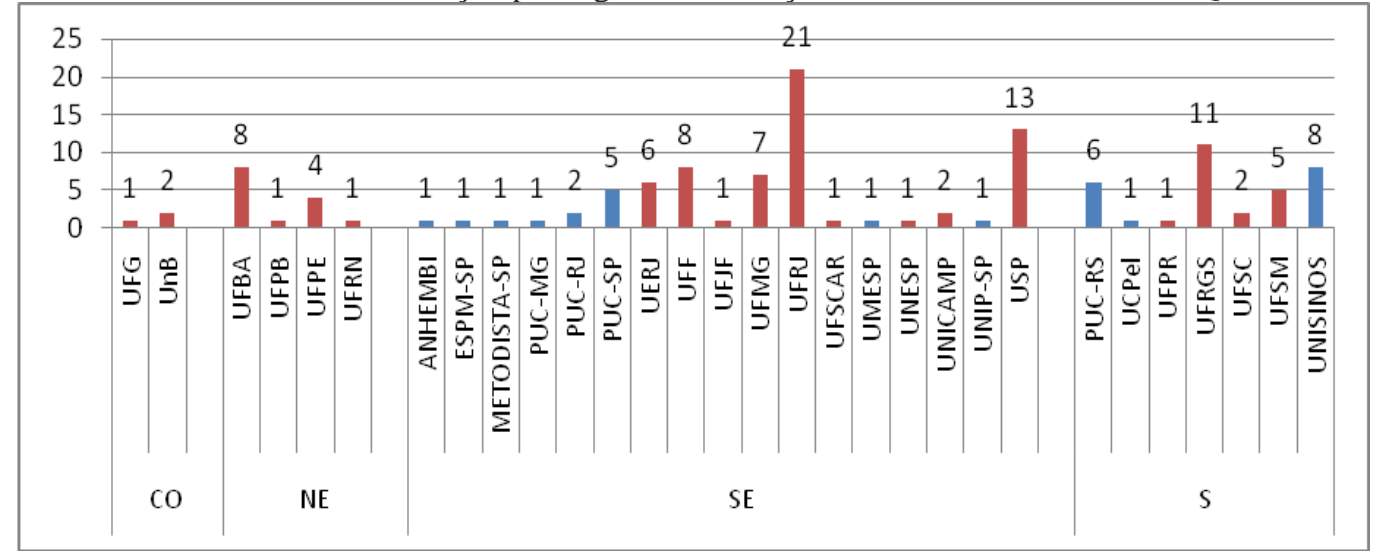

Fonte: Elaborado pelos autores.

Observa-se que existe uma concentração de bolsistas PQ nas regiões Sudeste e Sul, apresentando um percentual de $59 \%$ e $27 \%$, respectivamente. 
O Gráfico 2 também aponta a denominação das universidades que a esses bolsistas estão vinculados. A cor vermelha em destaque no Gráfico representa que a instituição é Pública; a cor azul em evidência na mesma representação indica que a instituição é Privada. Ao verificar o vínculo institucional, observa-se que os bolsistas PQ se concentram nas instituições públicas (77\%). Infere-se que seja devido ao fato de instituições públicas terem tradicionalmente perfil para desenvolvimentos de pesquisa. Segundo Viotti (2010), 90\% dos laboratórios e centros de pesquisa estão concentrados em universidades públicas, acarretando maior potencial de formação de recursos humanos em graduação e pós-graduação no país. É possível a interpretação de que a vinculação institucional do pesquisador com universidades públicas se configura um tipo de capital científico, afinal, estas têm "consolidado a sua posição como locus de desenvolvimento de pesquisa científica e tecnológica no país" (CORREIA, 2012, p. 21). Salienta-se, porém, que o Brasil já dispõe de universidades privadas de referência e tradição em pesquisa.

O Gráfico 3 detalha, de forma específica, a distribuição de bolsas pelos grupos PQ 1 e PQ 2 nas regiões Sudeste, Sul, Nordeste e Centro-Oeste.

Gráfico 3 - Quantitativo de bolsas PQ 1 e PQ 2 por regiões.

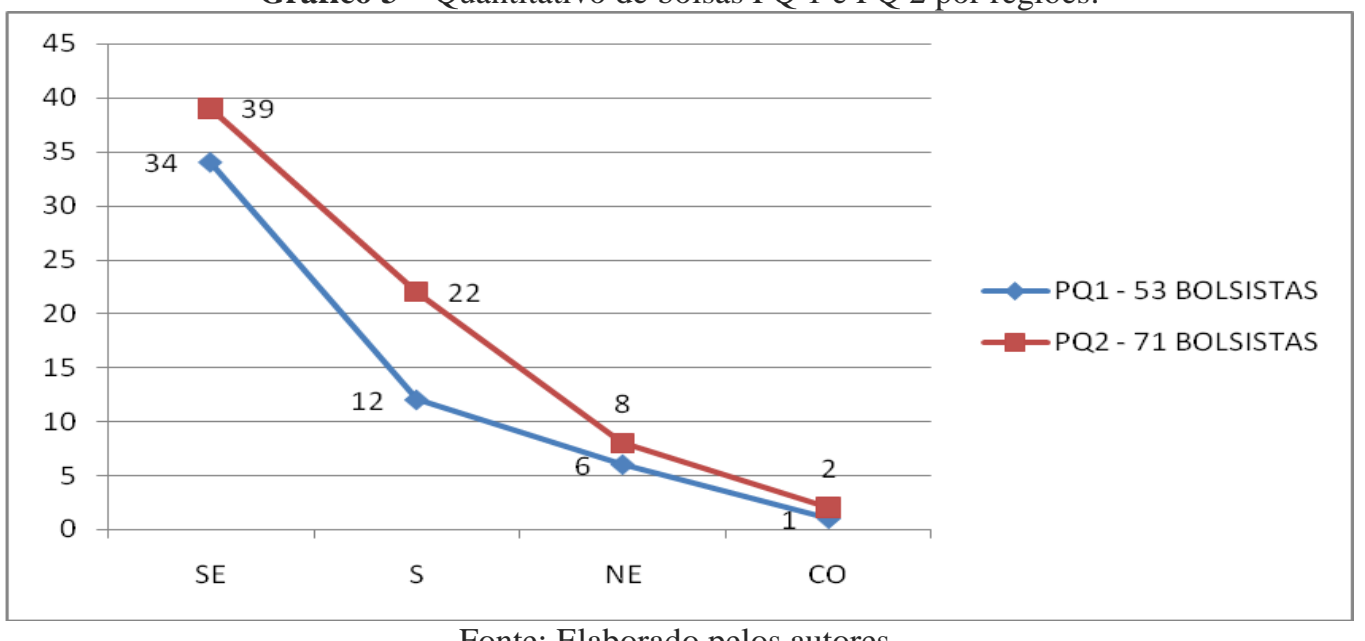

Fonte: Elaborado pelos autores.

Mais um gráfico que aponta a concentração de pesquisadores de alto nível em determinadas regiões (Sudeste e Sul). É notório que possa ocorrer uma tendência na concentração desses bolsistas devido ao elevado número de cursos de pós-graduação centrados nessas regiões. 
Observa-se, ainda, que a concessão de bolsas PQ está ligada não apenas à produtividade científica, mas também ao "reconhecimento internacional e participação efetiva na melhoria do campo científico-teórico da disciplina" (MELO, 2015, p. 75). Faz sentido imaginar que quaisquer concentrações de melhores condições para o desenvolvimento científico irão acarretar uma cultura de tradição de produtividade científica, realizando movimentos perenes. Sobre a leitura dos números do Gráfico 3 é possível recordar o Efeito Mateus, que, segundo Melo, indica que

[...] os números tendem a acarretar o vício do agraciamento para os pesquisadores detentores de grandes quantias de publicação e a censura para os menos favorecidos. Salienta-se que neste contexto não há como não levar em consideração o fato que o desenvolvimento da ciência é uma atividade que exige recursos, e estes são limitados pela política científica nacional. (MELO, 2015, p. 71).

No Gráfico 4 é descrito o tipo de produção dos bolsistas PQ 1 e PQ 2 (livro; capítulo de livro; trabalhos — anais de congresso; artigos em periódicos).

Gráfico 4 - Comparação entre os bolsistas PQ 1 e PQ 2: produção científica.

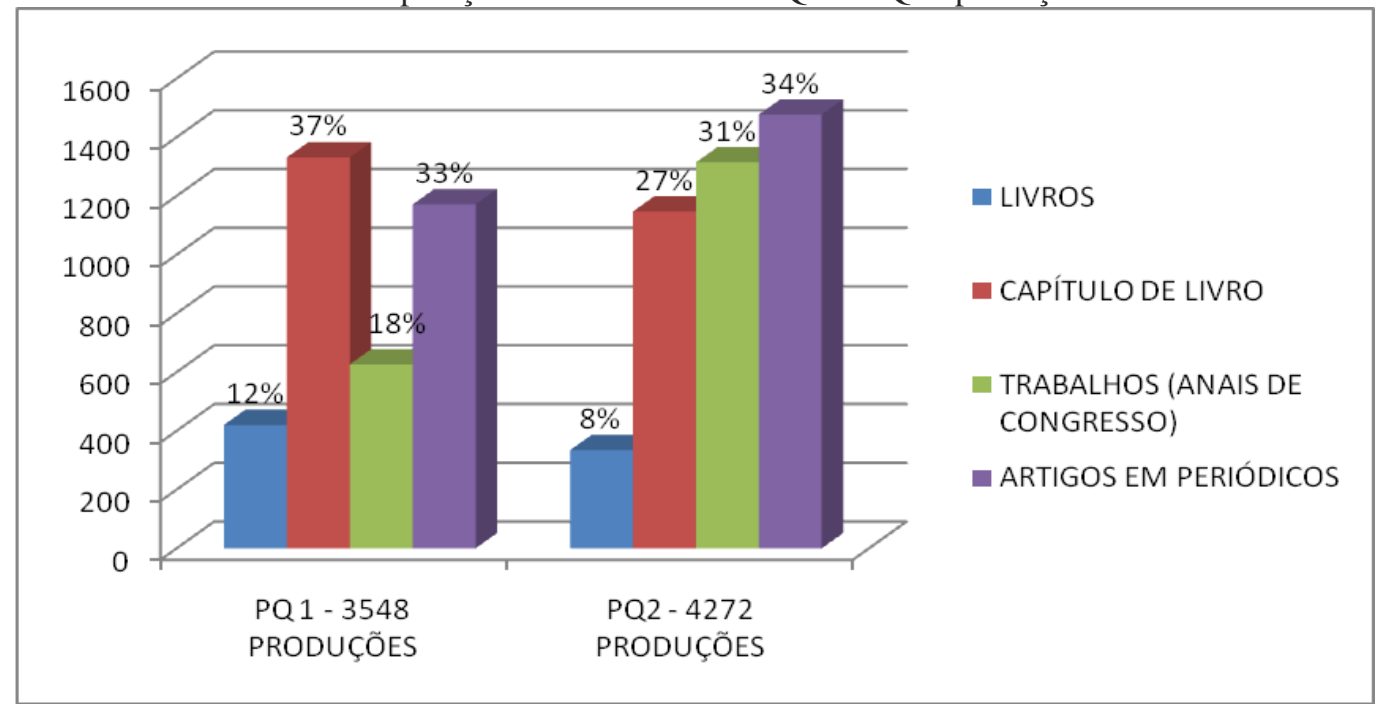

Fonte: Elaborado pelos autores.

É possível verificar que o maior grupo (PQ $2-71$ bolsistas) registrou maior produtividade (4.272). No entanto, o grupo PQ 2 apresenta menores índices em segmentos considerados estratégicos para avaliação periódica do $\mathrm{CNPq}$, como é o caso da produção de livros, capítulos de livros e artigos em periódicos. Ou seja, o grupo PQ 1 (53 bolsistas) produziu menos, mas produziu de maneira mais 
estratégica e organizada, satisfazendo as exigências da Resolução nº 016/2006 do CNPq (2006).

As Tabelas 1 e 2 demonstram o quantitativo dos artigos publicados, bem como o estrato Qualis das revistas escolhidas pelos pesquisadores para a submissão de seus trabalhos. A pesquisa teve como fonte um documento (datado de 12 jan 2015) do Sistema WebQualis com indicativos de estratos dos periódicos científicos cadastrados na área de Ciência Sociais Aplicadas I. Atualmente o WebQualis foi substituído pela Plataforma Sucupira. O motivo da não utilização da Plataforma Sucupira da CAPES foi que, até a data final de confecção deste documento, existia um funcionamento errático no sistema de busca pelo estrato dos periódicos. Tanto o antigo Sistema WebQualis quanto a atual Plataforma Sucupira são indicadores de qualidade dos meios responsáveis pela comunicação científica formal em periódicos.

Tabela 1 - Quantitativos de artigos publicados por bolsistas PQ 1 e estrato Qualis: Regiões. SUDESTE SUL NORDESTE CENTRO- TOTAL

OESTE

\begin{tabular}{l|cc|cc|cc|cc|cc}
\hline & Quant & $\%$ & Quant & $\%$ & Quant & $\%$ & Quant & $\%$ & Quant & $\%$ \\
\hline A1 & 14 & $\mathbf{2}$ & 18 & $\mathbf{7}$ & 7 & $\mathbf{6}$ & - & - & 39 & $\mathbf{4}$ \\
A2 & 153 & $\mathbf{2 0}$ & 58 & $\mathbf{2 3}$ & 25 & $\mathbf{2 0}$ & 2 & $\mathbf{1 8}$ & 238 & $\mathbf{2 0}$ \\
B1 & 221 & $\mathbf{2 9}$ & 97 & $\mathbf{3 8}$ & 51 & $\mathbf{4 0}$ & 6 & $\mathbf{5 1}$ & 375 & $\mathbf{3 2}$ \\
B2 & 64 & $\mathbf{8}$ & 11 & $\mathbf{4}$ & 8 & $\mathbf{6}$ & - & - & 83 & $\mathbf{7}$ \\
B3 & 41 & $\mathbf{5}$ & 16 & $\mathbf{6}$ & - & - & - & - & 57 & $\mathbf{5}$ \\
B4 & 57 & $\mathbf{7}$ & 14 & $\mathbf{5}$ & 7 & $\mathbf{5}$ & 1 & $\mathbf{9}$ & 79 & $\mathbf{7}$ \\
B5 & 45 & $\mathbf{6}$ & 12 & $\mathbf{5}$ & 3 & $\mathbf{2}$ & - & - & 60 & $\mathbf{5}$ \\
C & 45 & $\mathbf{6}$ & 7 & $\mathbf{3}$ & 9 & $\mathbf{7}$ & - & - & 61 & $\mathbf{5}$ \\
SEM & 135 & $\mathbf{1 7}$ & 24 & $\mathbf{9}$ & 18 & $\mathbf{1 4}$ & 2 & $\mathbf{1 8}$ & 179 & $\mathbf{1 5}$ \\
ESTRATO & & & & & & & & & & \\
\hline \multicolumn{1}{c}{ TOTAL } & $\mathbf{7 7 5}$ & $66 \%$ & $\mathbf{2 5 7}$ & $22 \%$ & $\mathbf{1 2 8}$ & $11 \%$ & $\mathbf{1 1}$ & $1 \%$ & $\mathbf{1 . 1 7 1}$ & $\mathbf{1 0 0 \%}$ \\
\hline
\end{tabular}

Fonte: Elaborado pelos autores.

Inicialmente, é perceptível na leitura das Tabelas 1 e 2 a gritante concentração da produtividade em periódicos científicos pelas regiões Sudeste e Sul. As duas regiões citadas registram, juntas, $88 \%$ das publicações no grupo PQ 1 e $89 \%$ no grupo PQ 2. São dados alarmantes, que demonstram complicações para as demais regiões que tentam consolidar uma cultura de produção científica reconhecida. 
É apresentado também um detalhamento da produtividade em revistas científicas. Os bolsistas PQ, como orienta a Resolução nº 016/2006 do CNPq (2006), devem contribuir para a melhoria do campo científico-teórico de sua área. Isso implica uma produção de alto nível, sugere também que a comunicação formal de seus trabalhos não aconteça em meios aleatórios de circulação científica.

Tabela 2 - Quantitativos de artigos publicados por bolsistas PQ 2 e estrato Qualis: Regiões.

\section{$\begin{array}{llll}\text { SUDESTE } & \text { SUL NORDESTE } & \text { CENTRO- } & \text { TOTAL }\end{array}$}

\begin{tabular}{|c|c|c|c|c|c|c|c|c|c|c|}
\hline & Quant & $\%$ & Quant & $\%$ & Quant & $\%$ & Quant & $\%$ & Quant & $\%$ \\
\hline A1 & 8 & 1 & 7 & 1 & - & - & - & - & 15 & 1 \\
\hline A2 & 154 & 20 & 91 & 17 & 17 & 13 & 1 & 3 & 268 & 18 \\
\hline B1 & 366 & 47 & 272 & 51 & 46 & 35 & 4 & 12 & 657 & 45 \\
\hline B2 & 55 & 7 & 31 & 6 & 6 & 4 & 1 & 3 & 92 & 6 \\
\hline B3 & 28 & 4 & 20 & 4 & 18 & 14 & 2 & 6 & 70 & 5 \\
\hline B4 & 40 & 5 & 25 & 5 & 20 & 15 & 10 & 30 & 121 & 8 \\
\hline B5 & 39 & 5 & 32 & 6 & 13 & 10 & 1 & 3 & 84 & 6 \\
\hline C & 26 & 3 & 14 & 3 & 3 & 2 & 2 & 6 & 45 & 3 \\
\hline $\begin{array}{l}\text { SEM } \\
\text { ESTRATO }\end{array}$ & 66 & 8 & 38 & 7 & 9 & 7 & 12 & 37 & 125 & 8 \\
\hline TOTAL & 782 & $53 \%$ & 530 & $36 \%$ & 132 & $9 \%$ & 33 & $2 \%$ & 1.477 & $100 \%$ \\
\hline
\end{tabular}

Possuir artigos publicados em periódicos que possuam indicadores de excelência é, sem dúvida alguma, um capital científico, uma espécie de moeda de troca importante no campo científico, em que fica evidenciada a luta por melhores posições e conquistas. Podem-se tomar como exemplo as publicações em revistas de estrato mais cobiçadas (A1, A2 e B1, respectivamente).

Os bolsistas PQ 1 possuem 56\% de suas publicações em revistas de estrato B1 (32\%), A2 (20\%) e A1 (4\%); os bolsistas PQ 2 concentram 64\% de suas produções em periódicos de estrato B1 (45\%) A2 (18\%) e A1 (1\%). Mesmo ambos possuindo uma concentração em revistas de estratos bem avaliados, existe no grupo PQ 1 maior equidade na destinação das referentes produções, enquanto no grupo PQ 2 existe um movimento centrípeto para publicação em periódicos de estrato B1. Outro detalhe no grupo PQ 1 é o alcance de 4\% de suas publicações em revistas de maior credibilidade e confiança (nacional e internacionalmente), segundo o indicador WebQualis. Esse detalhe é significativo para esse grupo e 
também, de forma individual, para os agentes responsáveis pelas referidas publicações (visto que no grupo PQ 1 existe uma subdivisão em: PQ 1A, PQ 1B, PQ 1C e PQ 1D).

\subsection{Emparelhando resultados}

Buscou-se neste estágio da pesquisa identificar a presença de padrões nesses grupos específicos. Para as análises, foram estabelecidos como parâmetro padrões de incidência $\geq 50 \%$ e $\leq 49 \%$, afinal, os dois grupos PQ possuem exigências de produtividade, vagas de bolsas limitadas, pressões devido a progressões, rebaixamento ou exclusão, necessidade de reconhecimento no campo científico (e, quando possível, em outros campos). Ao estarem inseridos em uma lógica relacional específica entre agentes/pares e instituições, os índices poderão indicar a presença, ou não, da repetição de comportamentos.

O método do emparelhamento pode ser entendido como um auxiliar de apreensão/representação da realidade, sendo necessário lembrar que a leitura desse objeto de estudo sofreu influências diretas da literatura sobre o campo científico de Bourdieu (1983; 2004; 2008). Apoiado nas considerações de Miranda (1999), Pinho (2009, p. 47, grifo do autor) indica que representações conseguem se aproximar do real e podem ter interpretações variadas; em outras palavras, representar é uma atividade "[...] de compreender a realidade, a ela necessita-se empreender uma expressão de racionalidade, associando princípios, categorias, procedimentos e normas, a fim de que essa atividade se torne estável”.

É descrito a seguir, por meio dos Quadros 1 e 2, o cenário alcançado diante das características do perfil do pesquisador, a instituição à qual ele está vinculado e sua produtividade. A perspectiva de campo científico, de Bourdieu (2004; 2008), é visualizada quando os números recebem uma interpretação com base na ideia de conquista de capital simbólico/científico em um contexto de luta presente no campo científico.

Considera-se que prováveis indícios valorizados pelo CNPq, para indicar progressão de bolsa PQ aos pesquisadores, estão localizados na parte superior 
esquerda da primeira coluna, que também indica a porcentagem demarcada pelo grupo de 53 bolsistas PQ 1 em Comunicação. A parte inferior esquerda da primeira coluna também indica características presentes, porém que provavelmente recebem menor valor de reconhecimento de capital simbólico/científico, tanto para o CNPq quanto para os próprios pares envolvidos nesse conjunto de relações.

Quadro 1 - Características apreendidas: resultados emparelhados (PQ 1).

\begin{tabular}{|c|c|c|}
\hline \multicolumn{3}{|c|}{ CAMPO CIENTÍFICO E A POLÍTICA CIENTÍFICA NACIONAL (CNPq) } \\
\hline & $\begin{array}{l}\text { PROGRESSÃo } \\
\text { (ganho de capital científico) }\end{array}$ & \\
\hline $\begin{array}{l}\text { ESTÁGIO PÓS-DOUTORAL: 79\% } \\
\text { PÓS-DOUTORES }\end{array}$ & \multirow{5}{*}{$\begin{array}{c}\text { CAPITAL } \\
\text { SIMBÓLICO/CIENTÍFICO E O } \\
\text { CONTEXO DA LUTA }\end{array}$} & \multirow{5}{*}{ MÉRITO RECONHECIDO } \\
\hline IES PÚBLICA: 74\% & & \\
\hline PQ 1A, 1B E 1C: $68 \%$ & & \\
\hline $\begin{array}{l}\text { CAPÍTULOS DE LIVROS E ARTIGOS } \\
\text { EM PERIÓDICOS: } 70 \%\end{array}$ & & \\
\hline $\begin{array}{l}\text { ARTIGOS EM REVISTAS A1, A2 E } \\
\text { B1: 56\% }\end{array}$ & & \\
\hline \multicolumn{3}{|c|}{$\begin{array}{l}\text { REBAIXAMENTO OU EXCLUSÃO } \\
\text { (perda ou estagnação de capital científico) }\end{array}$} \\
\hline $\begin{array}{l}\text { FORMAÇÃO ACADÊMICA: } 21 \% \\
\text { DOUTORES }\end{array}$ & \multirow{5}{*}{$\begin{array}{c}\text { CAPITAL } \\
\text { SIMBÓLICO/CIENTÍFICO E O } \\
\text { CONTEXTO DA LUTA }\end{array}$} & \multirow{5}{*}{$\begin{array}{c}\text { MENOR } \\
\text { RECONHECIMENTO }\end{array}$} \\
\hline PQ 1D: 32\% & & \\
\hline $\begin{array}{l}\text { PUBLICAÇÕES EM REVISTAS SEM } \\
\text { QUALIS: } 15 \%\end{array}$ & & \\
\hline $\begin{array}{l}\text { PUBLICAÇÕES EM REVISTAS COM } \\
\text { QUALIS B2-C: } 29 \%\end{array}$ & & \\
\hline $\begin{array}{l}\text { TRABALHOS EM ANAIS DE } \\
\text { CONGRESSO: } 18 \%\end{array}$ & & \\
\hline
\end{tabular}

Fonte: Elaborado pelos autores.

Com base nos números, a afirmação realizada tem fundamento no fato de que todas as características que atribuem mérito maior, que geram progressões, tiveram um índice alto (superior ou igual a $\geq 50 \%$ ). Existe maior procura pela adequação do perfil às características ali percebidas (pós-doutores; vinculação a IES públicas, PQ 1A, 1B e 1C), bem como aos tipos de produções científicas ali descritas (capítulos de livro; artigos em periódicos bem conceituados — A1, A2, B1). 
As características localizadas na parte inferior do Quadro representam, provavelmente, risco de rebaixamento ou exclusão para os pesquisadores que optam por esse tipo de produção (publicações em revistas de Qualis com pouca evidência ou até mesmo sem estrato).

O próprio reconhecimento do pesquisador PQ 1D não é bem definido pelo CNPq; no entanto, é possível assinalar que os mesmos podem ser apontados como uma espécie de "periferia": circulando o grupo dos mais conceituados de pesquisadores (1A, 1B e 1C), bem como o grupo de menor reconhecimento (PQ 2). Mais uma vez, é reafirmado que existe um limite de bolsas a serem concedidas, ou seja, a conquista de capital científico é realizada na busca do reconhecimento, na conquista de espaço dentro do campo. Com isso, se esse pensamento é fixado nos demais agentes que compõem o grupo, estará definido um contexto de luta.

Assim como o Quadro 1, são emparelhados os prováveis indícios valorizados pelo CNPq para indicar progressão aos 71 bolsistas PQ 2. A parte inferior esquerda da primeira coluna também indica características presentes, porém que provavelmente recebem menor valor de reconhecimento.

São perceptíveis características como: i) Estágio Pós-Doutoral (69\%); ii) Vínculo a instituições de ensino superior públicas (80\%); iii) Publicações no formato de Capítulos de Livros e Artigos em Periódicos (62\%). O Quadro 2 demonstra que os referidos dados são capazes de revelar parte da lógica presente no grupo PQ 2, um sistema funcional que torna os agentes dinâmicos ao processo de luta por representatividade (mérito reconhecido) em que existe, por parte do $\mathrm{CNPq}$, uma pressão pela produtividade e aprimoramento desses pesquisadores. 
Indicativos do campo científico: análise da produtividade dos bolsistas PQ 1 e PQ 2 em

Comunicação (2004-2013)

Willian Lima Melo, Anna Elizabeth Galvão Coutinho Correia

Quadro 2 - Características apreendidas: resultados emparelhados (PQ 2). CAMPO CIENTÍFICO E A POLÍTICA CIENTÍFICA NACIONAL (CNPq)

\begin{tabular}{|c|c|c|}
\hline \multicolumn{3}{|c|}{ CAMPO CIENTÍFICO E A POLÍTICA CIENTÍFICA NACIONAL (CNPq) } \\
\hline \multicolumn{3}{|c|}{$\begin{array}{l}\text { PROGRESSÃo } \\
\text { (ganho de capital científico) }\end{array}$} \\
\hline $\begin{array}{l}\text { ESTÁGIO PÓS-DOUTORAL: } 69 \% \\
\text { PÓS-DOUTORES }\end{array}$ & \multirow{4}{*}{$\begin{array}{c}\text { CAPITAL } \\
\text { SIMBÓLICO/CIENTÍFICO E O } \\
\text { CONTEXO DA LUTA }\end{array}$} & \multirow{4}{*}{ MÉRITO RECONHECIDO } \\
\hline IES PÚBLICA: $80 \%$ & & \\
\hline $\begin{array}{l}\text { CAPÍTULOS DE LIVROS E ARTIGOS } \\
\text { EM PERIÓDICOS: } 61 \%\end{array}$ & & \\
\hline $\begin{array}{l}\text { ARTIGOS EM REVISTAS A1, A2 E } \\
\text { B1: } 64 \%\end{array}$ & & \\
\hline \multicolumn{3}{|c|}{$\begin{array}{c}\text { ESTAGNAÇÃO (MANUTENÇÃO NO GRUPO PQ 2 - RISCO) } \\
\text { (perda ou estagnação de capital científico) }\end{array}$} \\
\hline $\begin{array}{l}\text { FORMAÇÃO ACADÊMICA: } 31 \% \\
\text { DOUTORES }\end{array}$ & \multirow{4}{*}{$\begin{array}{c}\text { CAPITAL } \\
\text { SIMBÓLICO/CIENTÍFICO E O } \\
\text { CONTEXTO DA LUTA }\end{array}$} & \multirow{4}{*}{$\begin{array}{c}\text { MENOR } \\
\text { RECONHECIMENTO }\end{array}$} \\
\hline $\begin{array}{l}\text { PUBLICAÇÕES EM REVISTAS SEM } \\
\text { QUALIS: } 8 \%\end{array}$ & & \\
\hline $\begin{array}{l}\text { PUBLICAÇÕES EM REVISTAS COM } \\
\text { QUALIS B2-C: } 28 \%\end{array}$ & & \\
\hline $\begin{array}{l}\text { TRABALHOS EM ANAIS DE } \\
\text { CONGRESSO: } 31 \%\end{array}$ & & \\
\hline
\end{tabular}

Fonte: Elaborado pelos autores.

Apresentadas as variações desses grupos, parte-se, com o auxílio do Quadro 3, para análise das oscilações desses números, verificando os pormenores necessários para a leitura dos mesmos.

Quadro 3 - Resultados emparelhados: PQ 1, PQ 2 e oscilações.

\begin{tabular}{|l|c|c|c|}
\hline CARACTERÍSTICAS PQ́RITO RECONHECIDO & PQ 2 & OSCILAÇÃO \\
\hline ESTÁGIO PÓS-DOUTORAL & $79 \%$ & $69 \%$ & $10 \%$ \\
\hline IES PÚBLICA & $74 \%$ & $80 \%$ & $6 \%$ \\
\hline LIVROS E ARTIGOS & $70 \%$ & $61 \%$ & $9 \%$ \\
\hline PUBLICAÇÃO EM REVISTA A1, A2, B1 & $56 \%$ & $64 \%$ & $8 \%$ \\
\hline \multicolumn{1}{|c|}{ MENOR RECONHECIMENTO } \\
\hline SEM ESTÁGIO PÓS-DOUTORAL & $21 \%$ & $31 \%$ & $10 \%$ \\
\hline PUBLICAÇÃO EM REVISTA SEM QUALIS & $15 \%$ & $8 \%$ & $7 \%$ \\
\hline PUBLICAÇÕES EM REVISTA B2-C & $29 \%$ & $28 \%$ & $9 \%$ \\
\hline PUBLICAÇÕES EM ANAIS DE CONGRESSO & $18 \%$ & $31 \%$ & $13 \%$ \\
\hline
\end{tabular}

Fonte: Elaborado pelos autores.

O grupo PQ 1, ao analisar as prováveis características que garantam mérito, demonstrou destaque. Em apenas dois itens (vinculação a IES Públicas e 
Publicações em Revistas A1, A2, B1) esse grupo mostrou resultados inferiores ao grupo PQ 2. Ressalta-se, porém, que: i) a oscilação de $6 \%$ no resultado sobre o levantamento sobre vinculação institucional foi a menor registrada e pode ser justificada pela diferença quantitativa de agentes que compõem cada grupo (PQ 1 - 53; PQ 2 - 71); ii) mesmo o grupo PQ 2 possuindo uma produtividade maior em revistas de estrato A1, A2 e B1, é necessário lembrar que existe uma concentração específica de publicações em revista de estrato B1 (dos 64\% de publicações em revistas A1, A2 e B1, 45\% são em revistas B1).

Mesmo diante do fato da existência da centralização de pesquisadores beneficiados com o Programa de Bolsa de Produtividade em algumas regiões, cabe afirmar, diante dos resultados dessa pesquisa, que o sistema de distribuição desses bolsistas nos grupos PQ 1 e PQ 2 acontece de forma minimamente justa.

O método do emparelhamento consegue demonstrar a possibilidade de mobilidade dos agentes envolvidos. Aponta ainda quais características devem ser seguidas ou reafirmadas pelos pesquisadores em seus respectivos grupos, bem como as que não demonstram tanto reconhecimento entre pares e instituições. Com isso, esse método consegue conferir credibilidade às análises.

\section{Considerações}

Estudos como esses são capazes de mapear um sistema de produtividade específico de determinados grupos de pesquisadores. Auxiliam nas proposições e tomada de decisões de agentes e instituições diretamente envolvidos no processo de produção e produtividade científica brasileira.

Ao discutir a atual política científica no Brasil, por meio do Programa de Produtividade em Pesquisa, o presente trabalho levanta considerações pertinentes sobre a cultura de produção científica. Considerações sobre centralização de bolsas e, consequente, elevada produtividade em determinadas regiões do país são tópicos aqui tratados devido à consideração de que auxílios como as bolsas PQ deveriam promover a ciência e procurar algo próximo a uma equidade na produção científica entre regiões, gerando uma tradição de produtividade de alto nível em instituições de ensino superior em todo Brasil. Mesmo considerando a 
importância de programas de auxílio como esses, faz-se necessária a leitura crítica dos números apresentados.

Tentou-se tornar ameno o tom das discussões do referencial teórico, visto que mesmo com alguns autores demonstrando a incompatibilidade de suas obras, o que foi perceptível foi uma complementaridade de ideias partindo de Merton (2013) e Shinn e Ragouet (2008), problematizando com Bourdieu (2004; 2008) e relacionando com a realidade da ciência e da política científica brasileira com Viotti (2010) e a Sociedade Brasileira Para o Progresso da Ciência (2011).

O presente trabalho insere na Ciência da Informação a discussão sobre a aplicabilidade de métodos não habituais de percepção de fenômenos e situações. O emparelhamento, como recurso auxiliar de análise, demonstra capacidade de representação de uma realidade existente ao trabalhar de modo relacional duas dimensões: a do objeto da pesquisa e a da teoria utilizada para compreender o fenômeno. A síntese resultante da relação apresentada será sempre uma variável, pois estará condicionada ao processamento textual dos aportes teóricos utilizados.

\section{Agradecimentos}

Ao Programa de Pós-Graduação da Universidade Federal de Pernambuco pela acolhida para o desenvolvimento deste estudo. Agradeço, pela cuidadosa apreciação, ao Prof. Fábio Mascarenhas, à Prof ${ }^{a}$. Isaltina Gomes e à Prof ${ }^{a}$. Leilah Bufrem. À Izadora García, por todas as contribuições durante o levantamento dos dados.

\section{Referências}

BOURDIEU, P. O campo científico. In: BOURDIEU, P.; ORTIZ, R. (Org.). Pierre Bourdieu: sociologia. Tradução Paula Montero e Alícia Auzmendi. São Paulo: Ática, 1983. p.122-155. 
BOURDIEU, P. Os usos sociais da ciência da ciência: por uma sociologia clínica do campo científico. Tradução Denice Barbara Catani. São Paulo: UNESP, 2004.

BOURDIEU, P. Para uma sociologia da ciência. Tradução Pedro Elói Duarte. Lisboa: Edições 70, 2008.

CONSELHO NACIONAL DE DESENVOLVIMENTO CIENTÍFICO E TECNOLÓGICO. Resolução RN no 016/2006, de 06 de julho de 2006. Bolsas individuais no país. Disponível em: 〈http://www.cnpq.br/web/guest/view//journal_content/56_INSTANCE_0oED/10157/100343\#16061>. Acesso em: 7 ago. 2014.

CORREIA, A. E. G. C. A influência exercida pelo sistema de avaliação da CAPES na produção científica dos programas de pós-graduação em Física. 2012. Tese (Doutorado em Ciência da Informação) - Programa de Pós-Graduação em Ciência da Informação, Universidade Federal de Minas Gerais, Belo Horizonte, 2012.

GUIMARÃES, J. A. C.; GRACIO, M. C. C.; MATOS, D. F. O. Produção científica de bolsistas pesquisa em Ciência da Informação do Conselho Nacional de Desenvolvimento Científico e Tecnológico $(\mathrm{CNPq})$ - um estudo com artigos de periódicos. Datagramazero, Rio de Janeiro, v. 15, n. 2, p. A05, 2014.

LAVILLE, C.; DIONNE, J. A construção do saber: manual de metodologia de pesquisa em ciências humanas. Tradução Heloísa Monteiro e Francisco Settineri. Porto Alegre: Artmed; Belo Horizonte: UFMG, 1999.

LÉVY, P. As tecnologias da inteligência: o futuro do pensamento na era da informática. Tradução Carlos Irineu da Costa. Rio de Janeiro: Editora 34, 1993.

MELO, W. M. Indicativos sobre o campo científico da comunicação: uma análise da produção científica dos bolsistas de produtividade em pesquisa entre os anos de 2004-2013. 2015. Dissertação (Mestrado em Ciência da Informação) Programa de Pós-Graduação em Ciência da Informação, Universidade Federal de Pernambuco, Recife, 2015.

MELO, W. L.; CORREA, A. E. G. C. A produção científica dos bolsistas de produtividade PQ 1 em Comunicação (2004-2013): indicativos sobre o campo científico. In: ENCONTRO NACIONAL DE PESQUISA EM CIÊNCIA DA INFORMAÇÃO, 16., 2015, João Pessoa. Anais... João Pessoa: ANCIB, 2015.

MELO, W. L.; CORREA, A. E. G. C. C. A produtividade dos bolsistas PQ 2 (2004-2013): possíveis leituras sobre o campo científico. In: ENCONTRO BRASILEIRO DE BIBLIOMETRIA E CIENTOMETRIA, 5., 2016, São Paulo. Anais... São Paulo: USP, 2016. p. A10. 
MERTON, R. K. A ciência e a estrutura social democrática. In: MERTON, R. K.; MARCOVICH, A.; SHINN, T. (Org.). Ensaios de sociologia da ciência. Tradução Sylvia Gemignani Garcia e Pablo Rubén Mariconda. São Paulo: Associação Filosófica Scientiae Studia; São Paulo: Editora 34, 2013. p. 181-198.

MIRANDA, M. L. C. A. A organização do conhecimento e seus paradigmas científicos: algumas questões epistemológicas. Informare: Cadernos do Programa de Pós-Graduação em Ciência da Informação, Rio de Janeiro, v. 5, n. 2, p. 64-77, 1999.

MOREL, R. L. M. A pesquisa científica e seus condicionamentos sociais. Rio de Janeiro: Achiamé, 1979.

PINHO, F. A. Fundamentos da organização e representação do conhecimento. Recife: Ed. Universitária da UFPE, 2009.

SHINN, T.; RAGOUET, P. Controvérsias sobre a ciência: por uma sociologia trasversalista da atividade científica. Tradução Pablo Rubén Mariconda e Sylvia Gemignani Garcia. São Paulo: Associação Filosófica Scientia Studia; São Paulo: Editora 34, 2008.

SOCIEDADE BRASILEIRA PARA O PROGRESSO DA CIÊNCIA. Ciência, tecnologia e inovação para um Brasil competitivo. São Paulo: SBPC, 2011.

VIOTTI, E. B. Doutores 2010: estudos da demografia da base técnico-científica brasileira. In: CENTRO DE GESTÃO E ESTUDOS ESTRATÉGICOS. Doutores 2010: estudos da demografia da base técnico-científica brasileira. Brasília: Centro de Gestão de Estudos Estratégicos, 2010. p.10-50.

\title{
Indicators of scientific field: analysis of PQ 1 and PQ 2 Communication fellows' productivity (2004-2013)
}

\begin{abstract}
A survey about 124 PQ communication fellows' productivity is presented, aiming to associate the 7,820 PQ1 and PQ2 fellow's articles, published between 2004-2013, to the communication scientific field's characterization in Brazil. The nature of the present research is qualitative and exploratory and the pattern matching method was used as a subsidiary analysis strategy. The indicators which were found helped to understand some elements of the communication scientific field. The composition of this field is originated from social relations between the agents and from the use of knowledge and recognition
\end{abstract}


of their productions acts, which strengthens the non-random feature of both agents and institution's acts.

Keywords: Communication. Productivity fellows. Higher education — Research. Sociology knowledge.

Recebido em: 18/09/2016

Aceito em: 08/11/2016 